\title{
Etude comparative de la séroprévalence des infections au VIH, VHB ET VHC chez les donneurs de sang en collecte fixe et mobile.
}

\section{Comparative study of the seroprevalence of HIV, HBV and HCV infections among blood donors in fixed and mobile collection.}

\author{
Traoré $\mathbf{H}^{1}$, Guitteye $\mathrm{H}^{1}$, Sangho $\mathrm{O}^{2}$, Diarra AB ${ }^{1}$, Cissé $\mathrm{M}^{\mathbf{1}}$, Ba $\mathrm{A}^{\mathbf{1}}$, Fomba $\mathrm{M}^{1}$, Maïga $\mathrm{B}^{1,2}$, Baby $\mathbf{M}^{\mathbf{1}}$. \\ 1 Centre National de Transfusion Sanguine (CNTS), BP E344, Bamako, Mali \\ 2 Faculté de Pharmacie (FAPH), Université des Sciences, des Techniques et des Technologies de Bamako, Bamako, \\ Mali.
}

Auteur correspondant : Dr Hassana GUITTEYE, CNTS Bamako email : guith2003@yahoo.fr

\section{Résumé}

Objectif : L'objectif était de comparer la séroprévalence du Virus de l'Immunodéficience Humaine (VIH) et des Virus des Hépatites B et C (VHB et VHC) chez les donneurs de sang prélevés lors des collectes mobiles et en collectes fixes au Centre National de Transfusion Sanguine de Bamako. Patients et méthodes: Il s'agissait d'une étude prospective transversale qui s'est déroulée sur 15mois à Bamako et environs. Un total de 6600 donneurs dont 3300 pour chaque type de cabines (mobile et fixe) ont été inclus en fonction de leur aptitude au don de sang. Les échantillons sanguins ont été testés par l'ELISA. Les données saisies sur EPI INFO 7.0, ont été analysées sur SPSS 20.0. Le test de $\chi 2$ a été utilisé pour comparer les proportions avec un seuil de significativité $\alpha$ fixé à 0,05 . L'étude a été menée en respectant les règles d'éthique liées à la recherche sur les sujets humains en vigueur en République du Mali. Résultats : Le sex-ratio était de 4,96 en faveur des hommes et l'âge moyen était de 30,03 \pm 9,04ans. Les prévalences du VIH, du VHB et du VHC étaient respectivement $2 \%, 18,1 \%$ et $3 \%$ chez l'ensemble des donneurs. Le sexe masculin et la tranche d'âge [18 - 25ans] étaient significativement les plus touchés par le VHB. La prévalence du VIH était significativement plus élevée en collecte mobile $(2,5 \%)$ qu'en centre fixe $(1,5 \%)$, p=0,003. Les prévalences du VHB et du VHC étaient égales pour les deux collectes, avec $18,2 \%$ et $3 \%$ pour les mobiles et $18 \%$ et $3 \%$ pour les fixes. Les coinfections étaient plus fréquentes en collecte mobile qu'en cabine fixe. Conclusion : La prévalence du VIH était élevée en collecte mobile qu'en fixe. Aucune différence de prévalences n'existait entre les deux types de collectes pour le VHB et le VHC. Afin de couvrir les besoins transfusionnels en qualité et en quantité suffisante un réajustement de stratégie semble plus que nécessaire. Cette nouvelle approche doit être focalisée sur la fidélisation des donneurs reçus aussi bien en cabine fixe qu'en collecte mobile, indemnes de toutes pathologies transmissibles par transfusion sanguine.

Mots clés : cabine fixe, cabine mobile, don, séroprévalence, VIH, VHB, VHC

\begin{abstract}
Summary
Objective: The objective was to compare the seroprevalence of human immunodeficiency virus (HIV) and hepatitis B and $\mathrm{C}$ (HBV and HCV) viruses among blood donors seen in mobile and fixed booths at the National Blood Transfusion Center in Bamako. Patients and methods: This was a cross-sectional prospective study that took place over 15 months in Bamako and around. A total of 6,600 donors, including 3,300 for each type of collection (mobile and fixed), were included according to their ability to donate blood. Blood samples were tested by ELISA. The data entered on EPI INFO 7.0 was analyzed on SPSS 20.0. The $\chi^{2}$ test was used to compare proportions with a meaningful threshold of 0.05 . The study was carried out in accordance with the ethical rules related to research on human subjects. Results: The sex ratio was 4.96 and the average age was $30.03 \pm 9.04$ years. Prevalence of HIV, HBV and HCV were $2 \%, 18.1 \%$ and $3 \%$, respectively, among all donors. Male and age group [18 - 25] were significantly affected by HBV. HIV prevalence was significantly higher in mobile collection $(2.5 \%)$ than in fixed centers $(1.5 \%), \mathbf{p}=\mathbf{0 . 0 0 3}$. HBV and HCV prevalence were equal for both collections, with $18.2 \%$ and $3 \%$ for mobile booth and $18 \%$ and $3 \%$ for fixed ones. Coinfections were more common in mobile collection than in fixed ones. Conclusion: HIV prevalence was high in mobile rather than fixed collection. There was no difference in prevalence of HBV and HCV between the two types of collections. In order to cover transfusion needs in quantity and quality, a strategy adjustment seems more than necessary. This new approach must focus on customizing donors seen in both fixed and mobile booths, free of infections transmitted by transfusion.
\end{abstract}

Key words: Blood, Booth, donation, seroprevalence, HIV, HBV, HBV, Bamako

\section{Introduction}

Les infections dues aux Virus de l'Immunodéficience Humaine (VIH), de l'Hépatite B (VHB) et de l'Hépatite $\mathrm{C}$ (VHC) sont des problèmes majeurs de santé publique à travers le monde [1,2]. Les personnes infectées par le VHB ou le VHC sont exposés à un risque d'évolution vers le carcinome hépatocellulaire et/ou de cirrhose. Ce risque est considérable lorsqu'il existe une coinfection VIH-Hépatites B et/ou C. Le dépistage systématique de ces virus dans les dons de sang avant leur utilisation, est l'une des recommandations de l'Organisation Mondiale de la Santé (OMS). La recherche des marqueurs viraux chez les donneurs de sang permet d'écarter les produits sanguins contaminants en vue de réduire la transmission 
des marqueurs par transfusion aux receveurs [3]. Selon l'OMS, les prévalences du VIH, VHB et VHC chez les donneurs de sang dans les pays à revenu élevé sont respectivement de $0,003 \% ; 0,03 \%$ et $0,02 \%$ [4]. Celles des pays à faible revenu sont de 1,08\% (VIH), 3,70\% (VHB) et $1,03 \%$ (VHC) [4]. Une étude effectuée en France dans la région Nord Pas de Calais sur les contreindications au don de sang et des marqueurs biologiques en fonction des types de collecte, a montré que le taux de marqueurs viraux positifs est plus élevé dans les sites fixes et les entreprises et qu'il est plus bas dans les lycées et les universités [3]. En Afrique, plusieurs études de prévalences ont été menées sur les infections transmissibles par transfusion sanguine. Il existe peu de données sur ces infections dans les dons de sang issus des collectes mobiles. Au Congo le taux de séroprévalence dans les dons de sang était de 8,01\% pour le VHB suivi de ceux du VIH et du VHC respectivement de 2,67\% et 2,67\% [5]. Au Mali, Les prévalences VIH, VHB, et VHC chez les donneurs de sang étaient respectivement de $2,6 \% ; 13,9 \% ; 3,3 \%$ [6]. L'approvisionnement en produits sanguins est basé sur deux types de collectes : cabine fixe, et collecte mobile. Les collectes mobiles s'intensifient et aucune étude n'a été effectuée pour déterminer le taux des marqueurs positifs au niveau de ses différents sites. Si ces collectes s'avèrent être une source de donneurs présentant une séroprévalence moins élevée des infections au VIH, VHB et VHC alors plus d'efforts au niveau santé publique devront être consentis pour les optimiser au Mali.

\section{Méthodes}

Il s'agissait d'une étude prospective transversale conduite de septembre 2012 à décembre 2013. Le recrutement des donneurs s'effectuait en fonction de leur aptitude au don de sang sur les lieux de collectes, de façon systématique jusqu'à l'atteinte de la taille de l'échantillon qui était de 3300 par type de collecte. Les examens sérologiques ont été réalisés selon une technique immuno-enzymatique basée sur le principe en sandwich (l'ELISA combiné de 4ème génération pour le VIH et VHC). Cette technique a la particularité d'être plus sensible que les techniques de diagnostiques rapides usuelles. Elle permet de détecter l'infection à un stade plus précoce. Le principe est basé sur la recherche des marqueurs du VIH par le test Genscreen ${ }^{\text {TM }}$ ULTRA VIH Ag-Ab (Bio-rad, France) qui permet la détection des divers anticorps associés anti HIV-1 et/ou HIV-2 et de l'antigène p24 du virus. Les tests Monolisa ${ }^{\circledR}$ AgHBs ULTRA et Monolisa ${ }^{\circledR}$ Anti HCV plus version 2 (BIO-RAD) ont été utilisés respectivement pour la recherche de l'antigène HBs du VHB et de l'anticorps anti VHC. Seuls les cas indéterminés et positifs du VHB ont fait l'objet d'une confirmation à l'automate de sérologie Mini vidas ${ }^{\mathrm{TM}}$ (BIO MERIEUX).Les données recueillies ont été saisies sur EPI INFO 7.0 et analysées sur SPSS 20.0. Le test de $\chi^{2}$ a été utilisé pour comparer les proportions avec un seuil de significativité $\alpha$ fixé à 0,05 . Le protocole a bénéficié de l'approbation du comité d'éthique de l'Institut National de Recherche en
Santé Publique (INRSP). L'étude ne comportait aucun risque additionnel pour les participants. Pour le respect des données à caractères personnelles, les noms et prénoms n'ont pas été utilisés sur la fiche de collecte. Le numéro du don était utilisé pour identifier le donneur. Un consentement volontaire, libre et éclairé a été obtenu des participants avant leurs inclusions. L'étude s'est déroulée conformément aux règles d'éthique liées à la recherche sur les sujets humains en vigueur en République du Mali.

\section{Résultats}

Au total, 6600 donneurs ont été inclus dans notre étude. Aucun donneur n'a fait l'objet d'une double participation. Le sex ratio était de 4,96. L'âge moyen était de 30,03 ans \pm 9,04 ans avec des extrêmes [18 ans à 60 ans] (tableau I).

Tableau I : Données sociodémographiques des donneurs de sang de Bamako, $\mathrm{N}=6600$.

\begin{tabular}{lll}
\hline Caractéristiques & Effectif & \% \\
\hline Sexe & & \\
Féminin & 1106 & 16,8 \\
Masculin & 5494 & $\mathbf{8 3 , 2}$ \\
Tranche d'âge & & \\
$18-25$ & 2664 & $\mathbf{4 0 , 4}$ \\
$26-35$ & 2276 & 34,5 \\
$36-45$ & 1121 & 17,0 \\
$46-60$ & 539 & 8,2 \\
Niveau d'instruction & & \\
Non Scolarisé & 1008 & 15,3 \\
Primaire & 1225 & 18,6 \\
Secondaire & 1997 & $\mathbf{3 0 , 3}$ \\
Supérieur & 2370 & $\mathbf{3 5 , 9}$ \\
\hline
\end{tabular}

Plus de soixante un pourcent $(61 \%)$ des dons ont été effectués par les donneurs bénévoles volontaires (tableau II).

Tableau II : Caractéristiques du don de sang de Bamako, $\mathrm{N}=6600$.

\begin{tabular}{lll}
\hline Caractéristiques & Effectif & $\mathbf{\%}$ \\
\hline Types de don & & \\
$\quad$ Dons de compensation & 2541 & 38,5 \\
$\quad$ Dons bénévoles & 4059 & $\mathbf{6 1 , 5}$ \\
Types de sites & & \\
Associations & 809 & 24,5 \\
Confessions religieuses & 542 & 16,4 \\
Entreprises & 388 & 11,7 \\
Etablissements d'enseignement & 1036 & $\mathbf{3 1 , 4}$ \\
Administration judiciaire & 218 & 6,6 \\
Garnisons & 242 & 7,3 \\
Autres & 65 & 2,0 \\
\hline
\end{tabular}


Les prévalences des infections à VIH, VHB et VHC chez les donneurs de sang étaient respectivement $2 \%$, $18,1 \%$ et $3 \%$ (tableau III).

Tableau III : Séroprévalence des marqueurs viraux chez les donneurs de sang à Bamako, $\mathrm{N}=6600$.

\begin{tabular}{lcc}
\hline Marqueurs viraux & Positif & \% \\
\hline VIH & 130 & $\mathbf{2 , 0}$ \\
VHB & 1196 & $\mathbf{1 8 , 1}$ \\
VHC & 199 & $\mathbf{3 , 0}$ \\
Coinfections & & \\
VIH-VHB & 38 & 0,5 \\
VIH-VHC & 9 & 0,1 \\
VHB-VHC & 36 & 0,5 \\
VIH-VHB-VHC & 4 & 0,06 \\
\hline
\end{tabular}

La prévalence des coinfections était de $0,5 \%$ pour VIHVHB, $0,1 \%$ pour VIH-VHC, $0,5 \%$ pour VHB-VHC et $0,06 \%$ pour VIH-VHB-VHC (tableau III). La prévalence du VIH était plus élevée chez les hommes et dans la tranche 36-45ans (tableau IV). Il existait un lien statistiquement significatif entre l'infection à VIH et le type de collecte, $\mathrm{p}=0,003$ (figure I, tableau IV).

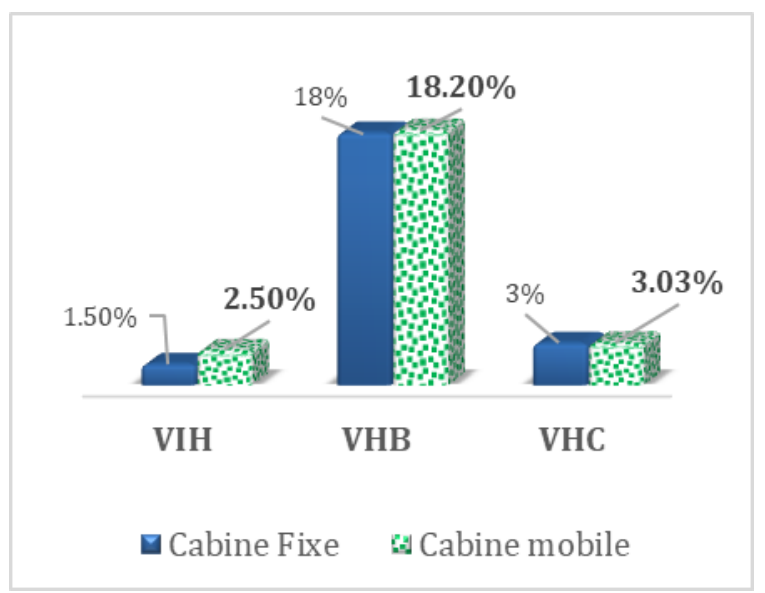

$\underline{\text { Figure } 1}$ : Comparaison entre marqueurs viraux et types de collecte.

Il existait un lien statistiquement significatif entre le type de donneur et les infections à VIH $(\mathrm{p}=0,003)$ et à VHB $(p=0,001)$ (tableau IV). La prévalence du VHB était significativement plus élevée chez les hommes ( $\mathrm{p}=$ $0,001)$, dans la tranche $18-25$ ans $(\mathrm{p}=0,047)$ et les primo donneurs $(p=0,001)$ (tableau IV). La plus forte prévalence observée lors des collectes était de $24,5 \%$ pour le VHB parmi les associations et groupements $(\mathrm{p}=0,002)$ (tableau IV). La prévalence du VHC était significativement plus élevée dans les Entreprises, $\mathrm{p}=0,006$ que dans les autres sites (tableau IV).

\section{Discussion}

Les analyses sanguines ont été réalisées conformément aux recommandations du fabricant. Les échantillons sanguins positifs aux VIH et VHC n'ont pas été confirmés. Les donneurs chez qui ces échantillons provenaient ont été conseillés de prendre contact avec un centre de dépistage pour confirmer leur statut sérologique. Afin d'assurer la sécurité du receveur, les poches de sang issues de ces donneurs étaient systématiquement éliminées. Hormis l'Ag HBs, les autres marqueurs du VHB n'ont pas été déterminés. Les échantillons et les poches contaminés ont été détruits conformément aux procédures du manuel de gestion des déchets biomédicaux. Les bonnes pratiques de laboratoire ont été scrupuleusement observées. Les participants déclarés positifs aux marqueurs viraux étaient orientés vers un service spécialisé dans la prise en charge de ces pathologies. Les donneurs étaient majoritairement constitués d'hommes avec un sex ratio de près de 5 . Des résultats similaires ont également été observés au Cameroun [9] et en Côte d'ivoire [7]. Cette prédominance masculine pourrait s'expliquer par la présence de certaines contre-indications spécifiques à la femme: la grossesse, l'accouchement, l'allaitement moins de 06 mois et la période menstruelle. La tranche d'âge de 18-25ans était majoritairement représentée. Ceci était probablement dû aux multiples campagnes de sensibilisation et de promotion du don de sang effectuées dans les établissements d'enseignement secondaire et supérieur. Est considéré comme bénévole, toute personne ayant acceptée de faire un don par altruisme. Les dons de sang issus de cette catégorie de donneurs étaient de $61,5 \%$. Ce résultat contredit celui de la base des données du CNTS dans laquelle plus de 2/3 des poches de sang collectées au Mali provenaient des dons familiaux. Cette discordance s'expliquerait par le fait que tous les donneurs recrutés lors des collectes mobiles, étaient considérés comme des bénévoles. La séroprévalence VIH était de $2 \%$ chez les donneurs. Cette prévalence était en baisse comparativement aux résultats des travaux effectués antérieurement au CNTS $[5,6]$. Si le VIH a été isolé dans la plupart des liquides sécrétés par l'Homme, seul le sang, le sperme, les sécrétions cervico-vaginales et lait maternel ont été incriminés dans la transmission de cette infection. En transfusion, la fenêtre sérologique est un facteur pouvant accroitre le risque de transmission de ce virus. Il s'agit d'une période s'écoulant entre le contact avec le virus et la détection des premiers anticorps dans le liquide biologique. Avec les Tests de Diagnostic Rapide (TDR) cette fenêtre sérologique est plus longue, comparés à l'ELISA combiné de $4^{\text {ème }}$ génération qui permet de la réduire à 10jours. Des prévalences plus élevées ont été observées au Nigeria [13], au Mozambique [22] et au Kenya [23]. Elle était significativement plus élevée chez les primo donneurs. Ce résultat était superposable à celui issu d'étude effectuée au Burkina Faso [13] par contre inferieur aux résultats observés au Congo [18] et au Cameroun [10]. L'utilisation du don de sang comme moyen de dépistage était une pratique courante. Bon nombre de personnes effectuent un don, non pas par altruisme mais dans le but de connaitre leur statut sérologique [13]. Les hommes étaient les plus touchés. Des prédominances 
féminines du VIH ont été rapportées par certains auteurs africains [8, 17]. La séroprévalence du VIH était significativement plus élevée parmi les dons issus des collectes mobiles comparée à celles fixes. La plus forte prévalence a été observée dans les garnisons et la plus faible dans l'administration judiciaire. La séroprévalence du VHB était de 18,1\%. Cette infection prédominait de façon significative chez les jeunes de 18-25 ans et les hommes. De fortes prévalences ont été également observées chez les jeunes hommes au Mali [6] et au Nigeria [12]. Suite à la recommandation de l'OMS de vacciner les nouveaux nés dès la naissance, le Mali a introduit en 2000, le vaccin anti-hépatite B dans le programme élargi de vaccination. La recherche de l'Ag HBs est réalisée systématiquement chez les femmes enceintes à travers le bilan prénatal. Les primodonneurs étaient les plus infectés $(20,1 \%)$ avec une différence significative. Si un résultat comparable a été observé au Congo [8], il diffère cependant de ceux observés au Cameroun [9] et au Burkina Faso [13] où de fortes prévalences ont été rapportées chez les donneurs de compensation. La séroprévalence du VHB observée en cabine fixe était similaire à celle de la collecte mobile. Au cours de cette dernière, elle était significativement plus élevée parmi les dons issus des associations. En Côte d'ivoire [7], l'infection à VHB était plus élevée en collecte mobile qu'en cabine fixe. Quant au VHC, une séroprévalence 3\% a été observée. Des taux supérieurs ont été rapportés au Cameroun [10] et Nigeria [12] avec respectivement $4,8 \%$ et $6 \%$. Cette prévalence était en constante augmentation au Mali chez les donneurs de sang depuis 2004, causant ainsi un sérieux problème d'approvisionnement en produits sanguins labiles. Certains auteurs ont rapporté des taux inférieurs aux nôtres $[11,13]$. Le sexe masculin et la tranche d'âge 36-45ans étaient les plus touchés. Une prédominance de cette infection a été observée chez les moins de 20 ans et les plus de 40 ans au Burkina Faso CONCLUSION :

La prévalence du virus de l'immunodéficience humaine était élevée en collecte mobile qu'en cabine fixe. Aucune différence de prévalence n'a été constatée pour les virus des hépatites $\mathrm{B}$ et $\mathrm{C}$ aussi bien en collecte mobile et en cabine fixe. Les coinfections étaient plus fréquentes en collecte mobile qu'en cabine fixe. Au vue de tout ce qui précède, afin de couvrir les besoins transfusionnels en qualité et en quantité suffisante, un réajustement de stratégie semble plus que nécessaire. Cette nouvelle approche doit être focalisée sur la fidélisation des donneurs, reçus aussi bien en cabine fixe qu'en collecte mobile, indemnes de toutes pathologies transmissibles par transfusion sanguine. Des progrès considérables ont été obtenus dans la maîtrise du risque de transmission transfusionnelle de ces virus mais l'accent doit être mis sur la sensibilisation et la prévention dans la population en général et chez les donneurs en particulier.

\section{Conflit d'intérêt :}

Les auteurs ne déclarent aucun conflit d'intérêt pour ce travail.
[14]. D'un point de vue général, ceci semble être problématique en Afrique subsaharienne où les nouveaux antiviraux efficaces sur le virus de l'hépatite $\mathrm{C}$ sont quasi inaccessibles. Aucune différence de prévalence du VHC aussi bien en cabine mobile qu'en collecte fixe n'a été constatée. Par contre en collecte mobile, la plus forte prévalence a été observée dans l'administration judiciaire. Tous les cas de figures de coinfections VIH et hépatites $\mathrm{B}$ et $\mathrm{C}$ ont été observés. Ceci était un facteur intéressant du point de vue épidémiologique mais inquiétant sur le plan sécurité transfusionnelle. Des taux supérieurs aux nôtres ont été rapportés par certains auteurs au Cameroun $[9,19]$ et au Mali [6] (VIH-VHB); en Namibie [16] (VIH-VHC et VHB-VHC) et au Ghana [15] (VHB-VHC). Si les fréquences de ces coinfections sont importantes en Afrique subsaharienne, elles demeurent relativement faibles au Maroc [20], en Lybie [21]. Les trois marqueurs viraux étaient présents chez quatre donneurs, soit $0,06 \%$. Il existait peu de données dans la littérature sur cette triple coinfection chez les donneurs de sang. Des taux inferieurs ont été rapportés chez cette population au Burkina Faso [17] et au Cameroun [19]. Ce taux élevé de cette coinfection pourrait mettre en cause la qualité de sélection médicale des donneurs. Cette étape de la chaine transfusionnelle consiste à identifier et à écarter les donneurs ayant des comportements à risque. La multiplicité des partenaires sexuelles, l'usage des drogues par voie intraveineuse, les tatouages et les piercings sont des comportements susceptibles de majorer le risque de la transmission de ces virus. Une sélection médicale bien conduite associée à la technique ELISA pourrait réduire le taux de ces infections. En somme, ces coinfections étaient beaucoup plus fréquentes en collecte mobile qu'en cabine fixe et cela était probablement corrélée à la positivité du VIH plus élevée en collecte mobile.

\section{Contributions des auteurs}

Dr Hassana GUITTEYE : Elaboration du protocole et soumission au comité d'éthique de l'INRSP; Dr Hamadi TRAORE : Collecte, analyse, interprétation des données et rédaction du manuscrit; Dr Oumar SANGHO : Correction du manuscrit, soumission à la revue scientifique.; Dr Amadou B DIARRA, Dr Alhassane BAH, Dr Moussa CISSE, Dr Minkoro FOMBA : relecture ; Pr Boubacar Maiga relecture ; Pr Mounirou BABY : Encadrement

\section{Remerciements :}

A l'Institut Pasteur de Paris pour avoir accepté de prendre une partie du financement de ce travail ;

Aux donneurs de sang qui ont bien voulu nous consacré une partie de leur temps pour que nous puissions réaliser ce travail; Aux techniciens du laboratoire d'immunologie qui ont traité les échantillons conformément aux procédures.

Au CNTS pour avoir accepté l'étude au sein de la structure.

\section{REFERENCES}


1. Kilmarx PH. Global epidemiology of HIV. Curr Opin HIV AIDS. 2009 Jul;4(4):240-6.

2. Ott JJ, Stevens GA, Groeger J, Wiersma ST. Global epidemiology of hepatitis B virus infection : new estimates of age-specific $\mathrm{HBs} \mathrm{Ag}$ seroprevalence and endemicity. Vaccine. 2012;30(12):2212-9

3. Fontaine O, Villard F, Huart JJ. Etude des contreindications au don de sang et des marqueurs biologiques en fonction du type de collecte. Transf Clin Biol 1998; 5 (1).

4. Organisation Mondiale de la Santé. Sécurité transfusionnelle et approvisionnement en sang. Juin 2017.

https://www.who.int/fr/news-room/factsheets/detail/blood-safety-and-availability

5. Christian NK, Olivier M, Serge K, Michel MM et al. Profil épidémiologique et séroprévalence des donneurs de sang aux cliniques universitaires de Lubumbashi, République Démocratique du Congo. The Pan African Medical Journal. 2016; 23:175.

6. Diarra A, Kouriba B, Baby M, Murphy E, Lefrere J-J. HIV, HCV, HBV and syphilis Rate of positive donations among blood donations in Mali: lower rates volunteer blood donors. Transf Clin et Biol, 2009; 16; p444-7

7. Tounkara A, Sarro Y, Kristensen S, Dao S. et al. Seroprevalence of HIV/HBV coinfection in Malian blood donors. J Int Assoc Physicians AIDS Care 2009; 8: p 47-51

8. Kra O, N'dri N, Ehui E, Ouattara B, Bissagnene E. Prévalence de l'antigène HBs chez les donneurs de sang au centre régional de transfusion sanguine de Bouaké en 2001. Bull Soc Pathol Exot, 2007 ; 100 (2) : 127-9.

9. Namululi BA, Guerriei C, Dramaix M. Impact du mode de recrutement des donneurs de sang sur la prévalence $\mathrm{du}$ VIH et du VHB a' Bukavu, République démocratique du Congo. Med Sante Trop 2012 ; 22 (1) : 69-74

10. Noubiap JJN, Walburga Y, Joko A, Richie J et al. Seroepidemiology of human immunodeficiency virus, hepatitis $\mathrm{B}$ and $\mathrm{C}$ viruses, and syphilis infections among first-time blood donors in Edéa, Cameroon. Internal Journal of Infectious Diseases 2013; 17:832-7.

11. Dray X, Dray SR, Bronstein JA, Mattera D. Séroprévalence des virus de l'immunodéficience humaine et des hépatites $\mathrm{B}$ et $\mathrm{C}$ parmi les donneurs de sang en république de Djibouti. Med Trop 2005; 65 (1) : 39-42.

12. Tagny CT, Murphy EL, Lefrere JJ et coll. Groupe de recherches transfusionnelles d'Afrique francophone: bilan des cinq premières années. Transf Clin et Biol 2014 ; 21 (1) :37-42.

13. Buseri FI, Muhibi MA, Jeremiah ZA. Seroepidemiology of transfusion transmissible infectious diseases among blood donors in Osogbo, south-west Nigeria. Blood Transf 2009; 7: 293-9

14. Nébié KY, Olinger CM, Kafando E, Dahourou H, Diallo $\mathrm{S}$ et al. Faible niveau de connaissances des donneurs de sang au Burkina Faso, une entrave potentielle à la sécurité transfusionnelle. Transf Clin et Biol 2007 ; 14 : 446-52

15. Nkrumah B, Owusu M, Frempong H. O and Averu P. Hepatitis $\mathrm{b}$ and $\mathrm{c}$ viral infections among blood donors from rural Ghana. Ghana medical journal 2011; 45 (3): 97-100.

16. Mavenyengwa RT, Mukesi M, Chipare I and Shoombe E. Prevalence of human immunodeficiency virus, syphilis, hepatitis $\mathrm{B}$ and $\mathrm{C}$ in blood donations in Namibia. BMC Public Health 2014; 14: 424.

17. Nagalo MB, Sanou M, Bisseye C, Kabore MI et al. Seroprevalency of human immunodeficiency virus, hepatitis B and C viruses and syphilis among blood donors in Koudougou (Burkina Faso) in 2009. Blood Transfus 2011; 9:419-24

18. Mbendi NC, Longo MB, Mbendi NS, et al. Prévalence du VIH et de l'antigène HBS chez les donneurs du sang. Risque résiduel de contamination chez les receveurs de sang à Kinshasa-Est, République Démocratique du Congo. Med Trop $2001 ; 61$ : 139-42.

19. Fouelifack Y F, Keugoung B, Hortense FJ, Kouam N, Mendibi S, Dongtsa MJ. High Rates of Hepatitis B and $\mathrm{C}$ and HIV Infections among Blood Donors in Cameroon: A Proposed Blood Screening Algorithm for Blood Donors in Resource-Limited Settings. J Blood Transfus 2012; 2012:458372.

20. Jean U, HZ, Loubet U, Rachid H. Seroprevalence of viral markers among blood donors at the blood Center of Mohammed V Military Teaching hospital of Rabat, Morocco. Pan Afr Med J. 2016; 25:185.

21. Khmmaj A, Habas E, Azabi M. Frequency of hepatitis $\mathrm{B} \mathrm{C}$ and HIV viruses among blood donors in Libya. Libyan J Med 2010; 5:5333.

22. Jocelijn S, Phillipe G, Anja DW, Esther CC, Rosa M, Adelino JB, Ilhes VJ, Solon K, Carla DM, Jan J, Emmanuel B. Seroprevalence of transfusiontransmissible infections and evaluation of the prdonationscreening performance at the povincial Hospital Tete, Mozambique. BNC infectious diseases 2011; $11: 141$.

23. Kamande MW, Kibebe H, and Mokua J. Prevalence of Transfusion Transmissible Infections Among Blood Donated At Nyeri Satellite Transfusion Centre In Kenya. OSR Journal Of Pharmacy 2016; 6 :20-30. 
$\underline{\text { Tableau IV }}$ : Relations entre les marqueurs viraux et les caractéristiques sociodémographiques, $\mathrm{N}=6600$.

\begin{tabular}{|c|c|c|c|c|c|c|c|}
\hline Caractéristiques & Effectif & $\mathrm{VHI}+$ & $p$ & $V H B+$ & $p$ & $\mathrm{VHC}+$ & $p$ \\
\hline Sexe & & $\mathrm{n}(\%)$ & & $\mathrm{n}(\%)$ & & $\mathrm{n}(\%)$ & \\
\hline Féminin & 1106 & $17(1,5)$ & & $163(14,7)$ & 0,001 & & \\
\hline Masculin & 5494 & $113(2,1)$ & & $1033(\mathbf{1 8 , 8})$ & & $172(3,1)$ & \\
\hline \multicolumn{8}{|l|}{ Tranche d'âge } \\
\hline $18-25$ & 2664 & $48(1,8)$ & & $505(\mathbf{1 9}, 0)$ & 0,047 & $79(3,0)$ & \\
\hline $26-35$ & 2276 & $41(1,8)$ & & $418(18,4)$ & & $67(2,9)$ & \\
\hline $36-45$ & 1121 & $30(2,7)$ & & $198(17,7)$ & & $38(3,4)$ & \\
\hline $46-60$ & 539 & $11(2,0)$ & & $75(13,9)$ & & $15(2,8)$ & \\
\hline \multicolumn{8}{|l|}{ Type de donneur } \\
\hline $\begin{array}{l}\text { Don } \\
\text { compensation }\end{array}$ & 2541 & $42(1,7)$ & 0,003 & $505(19,9)$ & 0,001 & $73(2,9)$ & \\
\hline Primo-donneur & 2262 & $64(2,8)$ & & $455(\mathbf{2 0 , 1})$ & & $74(3,3)$ & \\
\hline Deuxième don & 638 & $8(1,3)$ & & $126(19,7)$ & & $25(3,9)$ & \\
\hline $\begin{array}{l}\text { Donneur } \\
\text { bénévole }\end{array}$ & 1159 & $16(1,4)$ & & $110(9,5)$ & & $27(2,3)$ & \\
\hline \multicolumn{8}{|l|}{ Type de collecte } \\
\hline Cabine fixe & 3300 & $48(1,5)$ & 0,003 & $595(18,0)$ & & $99(3,0)$ & \\
\hline Collecte mobile & 3300 & $82(2,5)$ & & $601(18,2)$ & & $100(3,0)$ & \\
\hline \multicolumn{8}{|l|}{ Type de sites } \\
\hline $\begin{array}{l}\text { Associations et } \\
\text { groupements }\end{array}$ & 809 & $14(1,7)$ & 0,001 & $198(24,5)$ & 0,002 & $19(2,3)$ & 0,006 \\
\hline $\begin{array}{l}\text { Confessions } \\
\text { religieuses }\end{array}$ & 542 & $18(3,3)$ & & $78(14,4)$ & & $17(3,1)$ & \\
\hline Entreprises & 388 & $18(4,6)$ & & $65(16,8)$ & & $17(4,4)$ & \\
\hline $\begin{array}{l}\text { Etablissements } \\
\text { d'enseignement }\end{array}$ & 1036 & $17(1,6)$ & & $186(18)$ & & $30(2,9)$ & \\
\hline $\begin{array}{l}\text { L'administratio } \\
n \text { judiciaire }\end{array}$ & 218 & $2(0,9)$ & & $36(16,5)$ & & $14(6,4)$ & \\
\hline Garnisons & 242 & $12(5,0)$ & & $26(10,7)$ & & $1(0,4)$ & \\
\hline Autres & 65 & $1(1,5)$ & & $12(18,5)$ & & $2(3,1)$ & \\
\hline
\end{tabular}

